\title{
Benign Breast Tumors among Senegalese Women: Diversity and Genetic Evolution of D-Loop
}

\author{
Daniel Doupa', Marc Noël Badji'1, Fatimata Mbaye',2, Sidy Ka ${ }^{3}$, Ahmadou Dem³, \\ Mamadou Kane ${ }^{2}$, Mbacké Sembène ${ }^{1,2}$ \\ ${ }^{1}$ Department of Biology Animal, Faculty of Science and Technology, University Cheikh Anta Diop, Dakar, \\ Senegal \\ ${ }^{2}$ Sudanian Sahel Animal Populations Biology (BIOPASS), Research Institute for Development (IRD), Bel-Air, \\ Senegal \\ ${ }^{3}$ Cancer Institut, Faculty of Medicine, Pharmacy and Odontology, Cheikh Anta Diop University, Dakar, Senegal \\ Email: badjimarcnoel@gmail.com
}

Received 25 July 2015; accepted 11 August 2015; published 20 August 2015

Copyright (C) 2015 by authors and OALib.

This work is licensed under the Creative Commons Attribution International License (CC BY). http://creativecommons.org/licenses/by/4.0/

(c) (i) Open Access

\section{Abstract}

Subsaharan Africa, as in Senegal, breast cancer is the second after that of cervical in women. However, although most of the studies on breast pathology for cancer, the overwhelming majority of breast lesions, palpable or not are benign and some of them can become cancerous. So this research is done to understand the impact of diversity and genetic evolution of the D-loop in benign breast lesions in senegalese women. The variability of the D-loop was investigated by PCR-sequencing, in twenty eight patients with benign breast tumor. The results revealed a significant presence of specific variants for breast benign tissue, as well as control tissues. The C150T mutation was associated with protection to the presence of benign breast tumors and G247A mutation implicated in an increased risk. Patients of mitochondrial haplogroup $L$ would be significantly more susceptible to these benign breast lesions. And the study of the genetic evolution of breast benign tumors revealed that the D-Loop is not under selection. Finally, a significant correlation was associated with haplotypes C309CC and witnessed the D310, which respectively constitute increased risk groups and susceptible to the contraction of benign breast lesions. All these results allowed to have a global view on the influence of pathogenic mutations on diversity and genetic evolution of the D-Loop observed in senegalese patients with benign breast tumor.

\section{Keywords}

Tumor, Benign, Breast Cancer, D-Loop, Diversity, Genetic Evolution, Senegal

Subject Areas: Genetics

How to cite this paper: Doupa, D., Badji, M.N., Mbaye, F., Ka, S., Dem, A., Kane, M. and Sembène, M. (2015) Benign Breast Tumors among Senegalese Women: Diversity and Genetic Evolution of D-Loop. Open Access Library Journal, 2: e1758. http://dx.doi.org/10.4236/oalib.1101758 


\section{Introduction}

In Subsaharian Africa, as in Senegal, breast cancer is the second cancer after that of the cervix in women [1]. This taboo being a wreaking scourge in Africa, is making its prognosis more difficult. Besides, his affections are generally associated with a fear of mutilation, loss of sexual attractiveness and death [2]. With all this information in the media, revealing among other things that one woman in eight will be infected during his life [3], one can easily understand that breast pathologies are a source of anxiety for many women, especially when their mammogram requires additional investigation. However, although most studies on breast cancer pathology concern the process, the overwhelming majority of breast lesions are benign or not palpable [3] [4].

In the body, cells accumulate over time mutations that can alter their behavior, especially their proliferation. Thus mutant cells can evolve and form tumors. Already in 1976, the oncologist Peter Nowell advanced the idea that tumors follow the laws of evolution discovered by Darwin. When most tumors are benign and go unnoticed, some, however, degenerate into cancer when growth becomes uncontrolled. The disease is even worse when the tumor cells, as a result of new mutations, become mobile and metastasize [5].

Benign tumors are not cancerous; they do not proliferate in the body and include a number of diverse and heterogeneous diseases whose nomenclature varies according to the authors [6]. Of these tumors we can distinguish those unrelated to breast cancer (gynecomastia, fibrotic disease) and benign breast diseases that can be associated to the cancer [7]. Among the most studied of the benign tumors, it can be cited: cysts, fibroadenomas, lipomas, intraductal papillomas or the phyllodes tumors. These last two may become cancerous tumors [8]. And one of the biggest challenges in the diagnosis and prevention of breast cancer is early and specific detection of the precancerous lesions.

It is in this context that fits the approach of population's genetic that can be very helpful in such a study of benign breast tumors, and in different ways. Through these multiple analytical tools, population's genetic allows among others to identify variants displaying an unusual level of genetic differentiation between human populations and to determine to what extent the different genomic order process, demographic and selective as well and socio-cultural forces involved in the initiation of tumor observed profiles. It can also help to better understand the evolution of a mutation that confers a greater risk of developing benign breast tumor and those involved in the development to cancer or those involved in resistance to these different pathologies, while completing the clinical and epidemiological approaches in the search for genes and polymorphisms involved in these human diseases.

Our understanding of the development of these tumors and the identification of mutations that determine the properties of the tumor cells are essential in order to reach unambiguous diagnoses or more targeted therapies.

So to better understand the implications of changes in the D-loop in diversity and the genetic evolution of benign breast tumors in the Senegalese population, this research has the following specific objectives: assess the diversity and genetic evolution of the D-loop in breast benign tumors and look for correlations between changes in the D-loop and benign breast tumors.

\section{Materials and Methods}

\subsection{Patients}

Thirty-one (31) Senegalese patients with benign breast tumor, supported by the Joliot Curie Cancerology Institute of Aristide Le Dantec hospital of Dakar, were the subject of this study. This study also included a group of 30 Senegalese women as controls.

The clinical and pathological data on the age of patients, the type, size of the tumor, as well as the location of benign breast tumors were obtained from clinical records. Thus patients whose age is known, are between 15 and 25 years, suffering for most of fibroadenoma (76.47\%) and samples were most often taken (42.11\%) in left breast or (36.84\%) in both breasts with a tumor size ranging between 0.5 and $3 \mathrm{~cm}$.

NB: All precautions to respect the anonymity and confidentiality of information are strictly followed and these data are made available only to the study personnel.

Following informed consent of patients, the study was approved by the ethics committee of the University Cheikh Anta Diop of Dakar.

\subsection{Extraction, Amplification, PCR and Sequencing}

DNA extractions mammary tissues were performed using two protocols: the Puregene DNA Purification method 
and Standard Qiagen method.

The extraction protocol previously described by Mbaye et al. in 2012 [9].

Polymerase chain reaction (PCR) allow to generate a considerable number of fragments and identical to the target sequence, here the D-loop, facilitating sequencing. This step is based on the ability of Taq polymerase to synthesize the complementary strand by using the matrix, with fragments of oligonucleotides complementary to the template strand, and thus serving as specific primers elongation ( $2.5 \mu \mathrm{l}$ of H408: TGTTAAAAGTGCATACCGCCA and $2.5 \mu \mathrm{l}$ of L16340: AGCCATTTACCGTACATAGCACA), and that in the presence of $2 \mu \mathrm{l}$ of dNTP (deoxyribonucleotide), 1 ul of additional $\mathrm{MgCl}_{2}, 5 \mu \mathrm{ml}$ of $10 \mathrm{X}$ buffer, and $34.9 \mu \mathrm{l}$ of Milli Q water until the synthesis double-stranded DNA. PCR was carried out either with a volume of $2 \mu$ lor concentrated DNA $4 \mu \mathrm{l}$ or with $8 \mu$ of DNA diluted in order to reach the optimum concentration.

This cyclical process is carried with the following amplification conditions: 15 min of denaturation at $95^{\circ} \mathrm{C}$ followed by 35 cycles, each cycle consisting of a denaturation (30 sec at $\left.95^{\circ} \mathrm{C}\right)$, hybridization $\left(30 \mathrm{sec}\right.$ at $62^{\circ} \mathrm{C}$ ) and elongation $\left(2 \mathrm{~min}\right.$ at $\left.72^{\circ} \mathrm{C}\right)$ followed by a final elongation period $\left(10 \mathrm{~min}\right.$ at $\left.72^{\circ} \mathrm{C}\right)$. At the end of the cycles, the amplification products are maintained in a temperature of $10^{\circ} \mathrm{C}$ in a thermocycler.

The D-loop was sequenced with primer L16340.

\subsection{Analysis}

The obtained sequences of TS and TB are cleaned and corrected using the v BioEdit software. 5.0.6 [10]. These steps permit to verify the correspondence between the chromatograms and the sequences of each individual to avoid any errors. Then these sequences are aligned using the ClustalW multiple editor Alignment [11] to highlight the similarities, letting then the position of insertions appear, deletions or substitutions.

The frequency distribution of the variants between TS and TB was studied according to the Fisher's exact test with the MitoTool software v. 1.1.2 [12] in order to see the different mutations of the D-loop conferring a greater risk of developing benign breast tumor and those involved in resistance to these various benign pathologies breast. To realize this, the sequences of TS and TB were compared to the revised Cambridge Reference Sequence (RCRS) of the MITOMAP [13] databank under No. NC_012920 accession gi: 251831106.

Then these sequences were ranked according to the haplogroups studied and compared to the controls in order to highlight the different haplogroups associated or not to the risk of developing benign breast tumor, with the Fisher exact test with MitoTool software v 1.1.2 [12]. These haplogroups divided into different families very common in certain geographic areas or even in some populations [14] are combinations of the various mutations in the DNA sequence (or haplotypes) grouped according to their phylogenetic relationships.

In order to study the genetic diversity of the D-loop between benign tumors and control tissues, the nucleotide sequences of compositions, their frequencies, as well as the nature of mutations and the report transversion/ transition were performed on MEGA6 [15].

Specific diversity of population parameters (FST) were calculated with Arlequin [16] and obtaining the genetic distances within and between populations (TS and TB) with MEGA 6 [15]. These parameters that reflect the differentiation and the structuring of the D-loop were made, initially for each population and then for different groups divided into individuals belonging or not to haplogroup Saharan L. The latter found to be the most common haplogroup in Senegal [17], as well as throughout West Africa [14].

To study the genetic evolution of the D-loop of benign breast tumors, the number of haplotypes, the diversity and divergence indices and neutrality tests and "Mismatch distributions" or number of pairwise differences were made with DnaSP [18]. These tests are intended to distinguish sequences which evolution follows a neutral progression model to those progressing in a nonrandom process.

Subsequently the study on the incidence of tumors was performed. First by a comparison between haplogroups and age of patients, and a comparison of these haplogroups compared to the typology of diagnosed benign tumors. It was followed by a statistical study based on the chi 2 test $(\chi 2)$, conducted using the XLSTAT-Pro 6.1.9 software [19]. Finally, a study was conducted of the frequency distribution of different haplotypes identified based on mutations revealed between positions 303 and 316 of mtDNA, as the Fisher exact test conducted using the MitoTool v 1.1.2 software [12].

For all statistical tests the level of significance (P-value) was chosen at $5 \%$, and the odds ratio which is interpreted as relative risk, was also carried out in some of these analyzes. To this end, the "risk factor" increases when studied Odds ratio is greater than 1 , while a preventive factor is rather noted an odds ratio less than. 


\section{Results}

\subsection{Nucleotide Sequences Obtained}

After alignment and correction and removal of sequences having a very large genetic difference, twenty-eight (28) of the thirty-one (31) sequences from benign tissue, with a length of $560 \mathrm{bp}$ were retained. To these were added twenty-nine (29) to the portion corresponding sequences of the D-loop studied, the same size, obtained from healthy breast tissue. Thus, in total fifty-seven (57) sequences will be considered in genetic analyzes.

\subsection{Variability and Structuring of the D-Loop}

\subsubsection{Mutations in the D-Loop}

The portion mtDNA studied extends from position 16374 to position 366, including all of the hypervariable region II (HV2) of the D-loop. The results of the search for mutations in patients and controls are summarized in Table 1.

The results obtained for the benign tissue (Table 1) indicate the presence of 72 variants with $58.33 \%$ of transitions, transversions of $36.11 \%$ and $5.56 \%$ of insertions against 62 variants for healthy tissue with fewer transitions (56.45\%) and more than transversions (43.55\%). However, these results show that there has 29 variants in common (VC) between healthy tissue (i.e. $46.77 \%$ of TS variants) and studied benign tissue (i.e. $40.28 \%$ of TB variants).

Among the most representative of the common variants (present in over $28 \%$ of the sequences studied) may be cited: G16390A; T16519C; A73G; T146C; T152C; T195C; A263G; C315CC, in addition to the C150T mutation which is present very significantly $(\mathrm{p}=1.0 \mathrm{E}-5)$ in healthy individuals $(41.38 \%)$ than in patients $(17.86 \%)$, unlike the mutation G247A more present significantly $(\mathrm{p}=0.0486)$ in patients $(28.57 \%)$ than in healthy individuals (10.34\%). The most represented specific variants to benign tissue are: G79T $(34.14 \%, \mathrm{p}=3.73 \mathrm{e}-08)$; G81T (39.29\%, $p=9.65 \mathrm{e}-10)$; G97A (46.43\%, $\mathrm{p}=2.75 \mathrm{e}-11)$; G228A (28.57\%, $\mathrm{p}=2.32 \mathrm{e}-07)$; T310C (35.71\%, $\mathrm{p}=6.72 \mathrm{e}-09)$ and healthy tissue: C16478A (10.34\%, $\mathrm{p}=0.0002)$; C151G $(10.34 \%, \mathrm{p}=0.0002)$; T204G $(13.79 \%, p=4.17 \mathrm{e}-6)$; G207A $(13.79 \%, \mathrm{p}=4.17 \mathrm{e}-6)$ with a very significant presence.

\subsubsection{Haplogroups of the D-Loop}

Of the fifty-seven (57) individuals sequenced a total of eight (8) haplogroups (B, D, H, L, M, T, U, X) was found. Only haplogroups L, U, B, and M are shown in patients, while haplogroups B, D, H, L, M, T and X are present in the controls (Table 2).

Haplogroup Saharan L, covers more than half of the individuals studied is $54.39 \%$, with a significant difference ( $\mathrm{p}=0.0104$; $\mathrm{OR}=2.1728)$ between benign tissue $(64.29 \%)$ and tissues healthy $(44.83 \%)$. Asian haplogroups B, D, and M followed with 28.07\%, and appear to be more present in patients (32.14\%) than in healthy subjects (24.14\%). Conversely, the European haplogroups and Berber origin (H, U, T, and X) are in much less very significantly $(\mathrm{p}=4.05 \mathrm{e}-07$; $\mathrm{OR}=0.0927)$ in patients $(3.57 \%)$ than in healthy Senegalese women where they are well represented with $31.03 \%$.

The analysis of haplogroups in the L line revealed the presence of L1 (with its sub branches L1c and L1b) that appears both in patients than in controls, unlike under haplogroup L0 only present significantly in patients (7 $14 \% ; \mathrm{p}=0.014)$. The sub haplogroup L2 alone occupies nearly $55 \%$ of the family L, $29.82 \%$ of the studied while under Senegalese Afro-Eurasian haplogroup L3 was observed at significant frequencies, with its subhaplogroups L3K and L3x for patients and L3e with a witness. Of these sub-branches of the family L, the most frequent in patients is L2a (21.43\%) with a moderately significant difference $(\mathrm{p}=0.052)$ compared with controls (10.34\%), while L2b is most present in controls $(13.79 \%)$ and revealed a significant difference $(p=0.0125)$ for patients with (3.57\%). The sub branches and B4a M6a Asian, observed only in patients are also well represented with $17.86 \%$, respectively $(\mathrm{p}=3.67 \mathrm{e}-06)$ and $10.71 \%(\mathrm{p}=0.0008)$. Among haplogroups in European and Berber origin, H1, H2 and T2 are observed and only significantly in the controls.

\subsubsection{Diversity DNA Sequences Benign Breast Tumors and Genetic Structure of Populations \\ 1) Genetic Diversity}

The 560 bp of the D-loop analyzed in the twenty-eight (28) patients suffering from benign tumors have $12.14 \%$ of variable sites and among them more than half, or $57.36 \%$ parsimony are informative, whereas for 
Table 1. Positions and frequencies of variants of the D-loop in benign tissue matched to control tissues.

\begin{tabular}{|c|c|c|c|c|c|c|c|c|c|c|c|}
\hline \multicolumn{5}{|c|}{ Mutations in the D-loop } & \multirow{2}{*}{$\begin{array}{l}\text { Listed on } \\
\text { Mitomap }\end{array}$} & \multicolumn{2}{|r|}{ Type of mutation } & \multicolumn{3}{|c|}{ Frequencies variants } & \multirow[b]{2}{*}{ OR } \\
\hline rCRS & Positions & VB & VC & VH & & Transition & Transversion Insertion & $\begin{array}{c}\text { HT } \\
(n=29)\end{array}$ & $\mathrm{BT}(\mathrm{n}=28)$ & P-Value & \\
\hline G & 16389 & A & & & + & $\mathrm{s}$ & & - & $3.57 \%$ & 0.1466 & - \\
\hline G & 16390 & & A & & + & s & & $27.59 \%$ & $35.71 \%$ & 0.7013 & 0.9081 \\
\hline $\mathrm{T}$ & 16392 & G & & & & & $\mathrm{v}$ & - & $7.14 \%$ & $* 0.0461$ & - \\
\hline $\mathrm{C}$ & 16393 & $\mathrm{~T}$ & & & + & $\mathrm{s}$ & & - & $7.14 \%$ & $* 0.0461$ & - \\
\hline $\mathrm{C}$ & 16395 & & & G & & & $\mathrm{v}$ & $3.45 \%$ & - & 0.0711 & - \\
\hline $\mathrm{C}$ & 16411 & & & A & & & $\mathrm{v}$ & $3.45 \%$ & - & 0.0711 & - \\
\hline A & 16421 & & & $\mathrm{C}$ & & & $\mathrm{v}$ & $3.45 \%$ & - & 0.0711 & - \\
\hline $\mathrm{T}$ & 16422 & & & $\mathrm{C}$ & + & $\mathrm{s}$ & & $3.45 \%$ & - & 0.0711 & - \\
\hline $\mathrm{T}$ & 16468 & & & G & & & $\mathrm{v}$ & $3.45 \%$ & - & 0.0711 & - \\
\hline $\mathrm{T}$ & 16469 & & & G & & & $\mathrm{v}$ & $6.90 \%$ & - & $* 0.0021$ & - \\
\hline G & 16477 & & & A & + & $\mathrm{s}$ & & $3.45 \%$ & - & 0.0711 & - \\
\hline C & 16478 & & & A & & & $\mathrm{v}$ & $10.34 \%$ & - & $* 0.0002$ & - \\
\hline $\mathrm{T}$ & 16479 & & & A & & & $\mathrm{v}$ & $3.45 \%$ & - & 0.0711 & - \\
\hline C & 16501 & & & G & & & $\mathrm{v}$ & $3.45 \%$ & - & 0.0711 & - \\
\hline $\mathrm{C}$ & 16511 & & & $\mathrm{~T}$ & + & $\mathrm{s}$ & & $3.45 \%$ & - & 0.0711 & - \\
\hline $\mathrm{T}$ & 16519 & & $\mathrm{C}$ & & + & $\mathrm{s}$ & & $65.52 \%$ & $60.71 \%$ & $* 0.0155$ & 0.6366 \\
\hline $\mathrm{T}$ & 16522 & & & A & & & $\mathrm{v}$ & $3.45 \%$ & - & 0.0711 & - \\
\hline A & 16525 & & & G & + & $\mathrm{s}$ & & $3.45 \%$ & - & 0.0711 & - \\
\hline C & 16527 & & $\mathrm{~T}$ & & + & $\mathrm{s}$ & & $6.90 \%$ & $14.29 \%$ & 0.5106 & 1.4215 \\
\hline $\mathrm{C}$ & 11 & & & A & & & $\mathrm{v}$ & $3.45 \%$ & - & 0.0711 & - \\
\hline $\mathrm{C}$ & 29 & & & A & + & & $\mathrm{v}$ & $3.45 \%$ & - & 0.0711 & - \\
\hline $\mathrm{T}$ & 55 & A & & & + & & $\mathrm{v}$ & - & $7.14 \%$ & $* 0.0461$ & - \\
\hline $\mathrm{C}$ & 33 & & & G & + & & $\mathrm{v}$ & $3.45 \%$ & - & 0.0711 & - \\
\hline C & 61 & & & $\mathrm{~T}$ & + & $\mathrm{s}$ & & $6.90 \%$ & - & $* 0.0021$ & - \\
\hline $\mathrm{C}$ & 64 & & $\mathrm{~T}$ & & + & $\mathrm{s}$ & & $6.90 \%$ & $7.14 \%$ & 0.5904 & 0.7067 \\
\hline A & 73 & & G & & + & $\mathrm{s}$ & & $93.10 \%$ & $92.86 \%$ & $* 0.0155$ & 0.6844 \\
\hline A & 77 & G & & & & $\mathrm{s}$ & & - & $10.71 \%$ & $* 0.0037$ & - \\
\hline G & 79 & $\mathrm{~T}$ & & & + & & $\mathrm{v}$ & - & $32.14 \%$ & $* 3.73 e^{-08}$ & - \\
\hline $\mathrm{C}$ & 80 & A & & & & $\mathrm{s}$ & & - & $14.29 \%$ & $* 0.0006$ & - \\
\hline G & 81 & $\mathrm{~T}$ & & & + & & $\mathrm{v}$ & - & $39.29 \%$ & $* 9.65 \mathrm{e}-10$ & - \\
\hline $\mathrm{T}$ & 83 & $\mathrm{C}$ & & & & $\mathrm{s}$ & & - & $3.57 \%$ & 0.1466 & - \\
\hline $\mathrm{C}$ & 86 & G & & & & & $\mathrm{v}$ & - & $17.86 \%$ & *0.0001 & - \\
\hline $\mathrm{T}$ & 89 & A & & & & & $\mathrm{v}$ & - & $21.43 \%$ & $* 1.59 \mathrm{e}-05$ & - \\
\hline
\end{tabular}




\section{Continued}

\begin{tabular}{|c|c|c|c|c|c|c|c|c|c|c|c|}
\hline G & 90 & $\mathrm{~T}$ & & & + & & $\mathrm{v}$ & - & $3.57 \%$ & 0.1466 & - \\
\hline G & 90 & A & & & + & s & & - & $7.14 \%$ & $* 0.0461$ & - \\
\hline C & 91 & A & & & & & $\mathrm{v}$ & - & $3.57 \%$ & 0.14661 & - \\
\hline $\mathrm{C}$ & 91 & G & & & + & & $\mathrm{v}$ & - & $17.86 \%$ & $* 0.0001$ & - \\
\hline A & 93 & & G & & + & $\mathrm{s}$ & & $17.24 \%$ & $17.86 \%$ & 0.3929 & 0.7463 \\
\hline A & 95 & & $\mathrm{C}$ & & + & & $\mathrm{v}$ & $10.34 \%$ & $3.57 \%$ & *0.0287 & 0.2810 \\
\hline C & 96 & $\mathrm{~T}$ & & & + & $\mathrm{s}$ & & - & $21.43 \%$ & $* 1.60 \mathrm{e}-05$ & - \\
\hline G & 97 & A & & & + & $\mathrm{s}$ & & - & $46.43 \%$ & $* 2.75 \mathrm{e}-11$ & - \\
\hline C & 98 & A & & & & & $\mathrm{v}$ & - & $3.57 \%$ & 0.1466 & - \\
\hline C & 114 & & & G & + & & $\mathrm{v}$ & $6.90 \%$ & - & *0.0021 & - \\
\hline C & 120 & & & A & & & $\mathrm{v}$ & $3.45 \%$ & - & 0.0711 & - \\
\hline $\mathrm{C}$ & 140 & A & & & & & $\mathrm{v}$ & - & $3.57 \%$ & 0.1466 & - \\
\hline G & 143 & & A & & + & $\mathrm{s}$ & & $10.34 \%$ & $17.86 \%$ & 0.5692 & 1.2791 \\
\hline $\mathrm{T}$ & 146 & & $\mathrm{C}$ & & + & $\mathrm{s}$ & & $27.59 \%$ & $32.14 \%$ & 0.4268 & 0.8044 \\
\hline C & 150 & & $\mathrm{~T}$ & & + & s & & $41.38 \%$ & $17.86 \%$ & $* 1.01 \mathrm{e}-05$ & 0.3007 \\
\hline C & 151 & & $\mathrm{~T}$ & & + & s & & $3.45 \%$ & $7.14 \%$ & 0.5371 & 1.6565 \\
\hline C & 151 & & & G & + & & $\mathrm{v}$ & $10.34 \%$ & - & $* 0.0002$ & - \\
\hline $\mathrm{T}$ & 152 & & $\mathrm{C}$ & & + & s & & $55.17 \%$ & $78.57 \%$ & 0.9281 & 1.0186 \\
\hline C & 182 & & $\mathrm{~T}$ & & + & $\mathrm{s}$ & & $27.59 \%$ & $28.57 \%$ & 0.2755 & 0.7272 \\
\hline A & 183 & & G & & + & s & & $3.45 \%$ & $3.57 \%$ & 1 & 0.9443 \\
\hline G & 185 & & A & & + & $\mathrm{s}$ & & $6.90 \%$ & $7.14 \%$ & 0.5904 & 0.7067 \\
\hline G & 185 & & $\mathrm{~T}$ & & + & & $\mathrm{v}$ & $6.90 \%$ & $14.29 \%$ & 0.5106 & 1.4215 \\
\hline G & 185 & & & $\mathrm{C}$ & + & & $\mathrm{v}$ & $3.45 \%$ & - & 0.0711 & - \\
\hline C & 186 & & A & & + & & $\mathrm{v}$ & $3.45 \%$ & $3.57 \%$ & 1 & 0.9443 \\
\hline A & 189 & & $\mathrm{C}$ & & + & & $\mathrm{v}$ & $3.45 \%$ & $3.57 \%$ & 1 & 0.9443 \\
\hline A & 189 & & G & & + & s & & $3.45 \%$ & $10.71 \%$ & 0.1746 & 2.6117 \\
\hline $\mathrm{T}$ & 195 & & $\mathrm{C}$ & & + & s & & $51.72 \%$ & $50.00 \%$ & 0.0502 & 0.6675 \\
\hline A & 197 & & & $\mathrm{C}$ & & & $\mathrm{v}$ & $3.45 \%$ & - & 0.0711 & - \\
\hline C & 198 & & $\mathrm{~T}$ & & + & $\mathrm{s}$ & & $24.14 \%$ & $14.29 \%$ & $* 0.0075$ & 0.4064 \\
\hline A & 200 & G & & & + & $\mathrm{s}$ & & - & $7.14 \%$ & $* 0.0461$ & - \\
\hline $\mathrm{T}$ & 204 & & $\mathrm{C}$ & & + & $\mathrm{s}$ & & $3.45 \%$ & $7.14 \%$ & 0.5371 & 1.6565 \\
\hline
\end{tabular}


D. Doupa et al.

\section{Continued}

\begin{tabular}{|c|c|c|c|c|c|c|c|c|c|c|c|c|}
\hline $\mathrm{T}$ & 204 & & & G & + & & $\mathrm{v}$ & & $13.79 \%$ & - & $* 4.17 \mathrm{e}^{-6}$ & - \\
\hline $\mathrm{T}$ & 206 & G & & & + & & $\mathrm{v}$ & & - & $3.57 \%$ & 0.1466 & - \\
\hline G & 207 & & & A & + & $\mathrm{s}$ & & & $13.79 \%$ & - & $* 4.17 \mathrm{e}-6$ & - \\
\hline $\mathrm{T}$ & 208 & G & & & + & & $\mathrm{v}$ & & - & $3.57 \%$ & 0.1466 & - \\
\hline $\mathrm{T}$ & 217 & A & & & & & $\mathrm{v}$ & & - & $3.57 \%$ & 0.1466 & - \\
\hline $\mathrm{T}$ & 217 & G & & & + & & $\mathrm{v}$ & & - & $3.57 \%$ & 0.1466 & - \\
\hline $\mathrm{T}$ & 220 & A & & & & & $\mathrm{v}$ & & - & $3.57 \%$ & 0.1466 & - \\
\hline G & 221 & A & & & & s & & & & $3.57 \%$ & 0.1466 & - \\
\hline G & 221 & & & $\mathrm{C}$ & + & & $\mathrm{v}$ & & $3.45 \%$ & - & 0.0711 & - \\
\hline C & 222 & $\mathrm{~T}$ & & & + & $\mathrm{s}$ & & & - & $10.71 \%$ & $* 0.0037$ & - \\
\hline $\mathrm{T}$ & 224 & G & & & & & $\mathrm{v}$ & & - & $3.57 \%$ & 0.1466 & - \\
\hline G & 228 & A & & & + & $\mathrm{s}$ & & & - & $28.57 \%$ & $* 2.32 \mathrm{e}-7$ & - \\
\hline C & 231 & A & & & & & $\mathrm{v}$ & & - & $7.14 \%$ & $* 0.0461$ & - \\
\hline A & 232 & & & $\mathrm{C}$ & & & $\mathrm{v}$ & & $3.45 \%$ & - & 0.0711 & - \\
\hline A & 235 & G & & & + & $\mathrm{s}$ & & & - & $3.57 \%$ & 0.1466 & - \\
\hline $\mathrm{T}$ & 236 & $\mathrm{C}$ & & & + & $\mathrm{s}$ & & & - & $7.14 \%$ & $* 0.0461$ & - \\
\hline A & 240 & & & $\mathrm{~T}$ & & & $\mathrm{v}$ & & $3.45 \%$ & - & 0.0711 & - \\
\hline G & 242 & A & & & & $\mathrm{s}$ & & & - & $3.45 \%$ & 0.2715 & - \\
\hline G & 247 & & A & & + & $\mathrm{s}$ & & & $10.34 \%$ & $28.57 \%$ & $* 0.0486$ & 2.0798 \\
\hline C & 256 & & & $\mathrm{~T}$ & + & $\mathrm{s}$ & & & $3.45 \%$ & - & 0.0711 & - \\
\hline A & 257 & $\mathrm{C}$ & & & & & $\mathrm{v}$ & & - & $3.45 \%$ & 0.2715 & - \\
\hline A & 263 & & G & & + & $\mathrm{s}$ & & & $100 \%$ & $100.0 \%$ & $* 0.0104$ & 0.6824 \\
\hline G & 275 & A & & & + & $\mathrm{s}$ & & & - & $7.14 \%$ & $* 0.0461$ & - \\
\hline $\mathrm{T}$ & 279 & & & $\mathrm{C}$ & + & $\mathrm{s}$ & & & $3.45 \%$ & - & 0.0711 & - \\
\hline $\mathrm{T}$ & 292 & & & A & + & & $\mathrm{v}$ & & $3.45 \%$ & - & 0.0711 & - \\
\hline A & 297 & & & G & + & $\mathrm{s}$ & & & $3.45 \%$ & - & 0.0711 & - \\
\hline C & 309 & & & $\mathrm{~T}$ & + & $\mathrm{s}$ & & & $3.45 \%$ & - & 0.0711 & - \\
\hline $\mathrm{T}$ & 310 & $\mathrm{C}$ & & & + & $\mathrm{s}$ & & & - & $35.71 \%$ & $* 6.72 \mathrm{e}-9$ & - \\
\hline $\mathrm{T}$ & 310 & TTC & & & + & & & i & - & $35.71 \%$ & $* 6.72 \mathrm{e}-9$ & - \\
\hline C & 311 & $\mathrm{~T}$ & & & + & $\mathrm{s}$ & & & - & $3.57 \%$ & 0.1466 & - \\
\hline C & 315 & & $\mathrm{CC}$ & & + & & & i & $93.10 \%$ & $60.71 \%$ & $* 8.16 \mathrm{e}^{-7}$ & 0.4365 \\
\hline C & 315 & CCG & & & + & & & $\mathrm{i}$ & - & $3.57 \%$ & 0.1466 & - \\
\hline G & 316 & & $\mathrm{C}$ & & + & & $\mathrm{v}$ & & $3.45 \%$ & $3.57 \%$ & 1 & 0.9443 \\
\hline G & 316 & & GA & & & & & $\mathrm{i}$ & $3.45 \%$ & $3.57 \%$ & 1 & 0.9443 \\
\hline C & 317 & $\mathrm{~T}$ & & & + & $\mathrm{s}$ & & & - & $3.57 \%$ & 0.1466 & - \\
\hline
\end{tabular}




\section{Continued}

\begin{tabular}{|c|c|c|c|c|c|c|c|c|c|c|c|c|}
\hline $\mathrm{C}$ & 320 & $\mathrm{~T}$ & & & + & $\mathrm{s}$ & & & - & $3.57 \%$ & 0.1466 & - \\
\hline $\mathrm{C}$ & 325 & & $\mathrm{~T}$ & & + & s & & & $3.45 \%$ & $7.14 \%$ & 0.5371 & 1.6565 \\
\hline $\mathrm{C}$ & 332 & & & $\mathrm{~T}$ & + & $\mathrm{s}$ & & & $3.45 \%$ & - & 0.0711 & - \\
\hline $\mathrm{C}$ & 332 & A & & & + & & $\mathrm{v}$ & & - & $3.57 \%$ & 0.1466 & - \\
\hline G & 347 & $\mathrm{C}$ & & & & & $\mathrm{v}$ & & - & $3.57 \%$ & 0.1466 & - \\
\hline A & 357 & & G & & + & $\mathrm{s}$ & & & $10.34 \%$ & $14.29 \%$ & 1 & 0.9916 \\
\hline \multicolumn{2}{|c|}{ Mutations } & 43 & 29 & 33 & $\begin{array}{c}73 \\
\text { (69.52\%) }\end{array}$ & $\begin{array}{c}53 \\
\text { (50.48\%) }\end{array}$ & $\begin{array}{c}48 \\
(45.72 \%)\end{array}$ & $\begin{array}{c}4 \\
(3.81 \%)\end{array}$ & \multirow{3}{*}{\multicolumn{4}{|c|}{ *: Significant $\mathrm{P}$-value, $\mathrm{p}<0.050$}} \\
\hline TB & 72 & $59.72 \%$ & $40.28 \%$ & - & $\begin{array}{c}\mathbf{5 3} \\
(73.61 \%)\end{array}$ & $\begin{array}{c}42 \\
\text { (58.33\%) }\end{array}$ & $\begin{array}{c}\mathbf{2 6} \\
(36.11 \%)\end{array}$ & $\begin{array}{c}\mathbf{4} \\
(5.56 \%)\end{array}$ & & & & \\
\hline TS & 62 & - & $46.77 \%$ & $53.23 \%$ & $\begin{array}{c}47 \\
(75.81 \%)\end{array}$ & $\begin{array}{c}\mathbf{3 5} \\
(56.45 \%)\end{array}$ & $\begin{array}{c}27 \\
(43.55 \%)\end{array}$ & - & & & & \\
\hline
\end{tabular}

Table 2. Frequency and diversity of haplogroups and sub-haplogroups found among Senegalese women.

\begin{tabular}{|c|c|c|c|c|c|c|c|c|c|c|c|c|}
\hline \multirow{2}{*}{$\begin{array}{l}\text { Distribution } \\
\text { areas }\end{array}$} & \multicolumn{2}{|c|}{ Haplogroups } & \multirow{2}{*}{$\begin{array}{c}\text { \%HT } \\
0.00\end{array}$} & \multirow{2}{*}{$\begin{array}{c}\text { \%BT } \\
7.14\end{array}$} & \multirow{2}{*}{$\begin{array}{l}\text { P-value } \\
*_{0.014}\end{array}$} & \multirow{2}{*}{$\begin{array}{c}\text { \%HT } \\
0.00\end{array}$} & \multirow{2}{*}{$\begin{array}{l}\% \text { BT } \\
7.14\end{array}$} & \multirow{2}{*}{$\begin{array}{l}\text { P-value } \\
* 0.0140\end{array}$} & \multirow[t]{2}{*}{$\% \mathrm{HT}$} & \multirow[t]{2}{*}{$\%$ BT } & \multirow[t]{2}{*}{ P-value } & \multirow[t]{2}{*}{ OR (95\% CI) } \\
\hline & Lo & L0a & & & & & & & & & & \\
\hline \multirow{8}{*}{ Sub-Saharan } & \multirow{2}{*}{ L1 } & L1b & 10.34 & 14.29 & 0.5181 & \multirow{2}{*}{13.79} & \multirow{2}{*}{17.86} & \multirow{2}{*}{0.5634} & \multirow{8}{*}{44.83} & \multirow{8}{*}{64.29} & \multirow{8}{*}{$* 0.0104$} & \multirow{8}{*}{$\begin{array}{c}2.1728 \\
(1.232-3.832\end{array}$} \\
\hline & & L1c & 3.45 & 3.57 & 1 & & & & & & & \\
\hline & & L2a & 10.34 & 21.43 & 0.0520 & & & & & & & \\
\hline & L2 & $\mathrm{L} 2 \mathrm{~b}$ & 13.79 & 3.57 & $* 0.0125$ & 27.59 & 32.14 & 0.6436 & & & & \\
\hline & & L2c & 3.45 & 7.14 & 0.3327 & & & & & & & \\
\hline & \multirow{3}{*}{ L3 } & L3e & 3.45 & 0.00 & 0.1140 & \multirow{3}{*}{3.45} & \multirow{3}{*}{7.14} & \multirow{3}{*}{0.3311} & & & & \\
\hline & & L3k & 0.00 & 3.57 & 0.1217 & & & & & & & \\
\hline & & L3x & 0.00 & 3.57 & 0.1217 & & & & & & & \\
\hline \multirow{6}{*}{ Asian } & B2 & B2g & 6.90 & 0.00 & *0.0058 & & & & \multirow{6}{*}{24.14} & \multirow{6}{*}{32.14} & \multirow{6}{*}{0.2702} & \multirow{6}{*}{$\begin{array}{c}1.4902 \\
(0.7999- \\
2.7763)\end{array}$} \\
\hline & & B4a & 0.00 & 17.86 & $* 3.67 \mathrm{e}^{-6}$ & 13.79 & 21.43 & 0.2640 & & & & \\
\hline & & B4c & 6.90 & 3.57 & 0.3640 & & & & & & & \\
\hline & D4 & $\mathrm{D} 4 \mathrm{j}$ & 3.45 & 0.00 & 0.1140 & 3.45 & 0.00 & 0.2462 & & & & \\
\hline & M6 & M6a & 0.00 & 10.71 & *0.0008 & \multirow{2}{*}{6.90} & \multirow{2}{*}{10.71} & & & & & \\
\hline & M56 & M56 & 6.90 & 0.00 & *0.0058 & & & 0.4595 & & & & \\
\hline & H1 & H1a & 6.90 & 0.00 & *0.0058 & 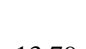 & 50 & - & & & & \\
\hline & H2 & $\mathrm{H} 2 \mathrm{a}$ & 6.90 & 0.00 & $* 0.0058$ & & & & & & & \\
\hline European & קT & $\mathrm{T} 2 \mathrm{~b}$ & 10.34 & 0.00 & $* 0.0006$ & 12 & ( & $* 7,100$ & 201010 & 257 & $* 10507$ & 0.0927 \\
\hline Berber & & $\mathrm{T} 2 \mathrm{c}$ & 3.45 & 0.00 & 0.1140 & & & & & & & $0.2748)$ \\
\hline & U5 & U5b & 0.00 & 3.57 & 0.1217 & 0.00 & 3.57 & 0.1212 & & & & \\
\hline & X3 & ХЗа & 3.45 & 0.00 & 0.1140 & 3.45 & 0.00 & 0.2462 & & & & \\
\hline
\end{tabular}

*: significant p-value. $\mathrm{p}<0.05$. 
twenty-nine (29) healthy controls (560 bp) $16.99 \%$ of sites are variable, with only $29.47 \%$ of parsimony informative sites. Thus the percentage of informative sites in parsimonies is higher in BD than in the TS. Another parameter tested is the nature of the mutations and the results obtained show that the level of the benign mammary tissues $32.24 \%$ of mutations are compared transversions to TS level where $30.99 \%$ are transversions. In all cases we will notice that the transitions are always the majority. This results in a transition/transversion report of 2.049 and 2.2267 for TB and TS respectively. The genetic diversity parameters of TS and TB are reported in Table 3.

Table 4 shows that frequency nucleotide bases $\mathrm{A}$ and $\mathrm{C}$ are more frequent with $30.12 \%$ and $27.69 \%$ respectively for TB and $30.01 \%$ and $27.44 \%$ for TS. It has the same torque $\mathrm{T}+\mathrm{C}$ which is higher than the combination $\mathrm{A}+\mathrm{G}$ for TB as with TS.

\section{2) Differentiation and Genetic Structure of Populations}

Genetic distances (d), which account for the genetic differences within and between tissues were calculated (Table 5), initially for each population and then for different groups divided into individuals belonging or not to haplogroup L. The genetic distance between benign tissues of Senegalese women studied (0.024) is slightly higher than that observed between healthy tissues $(0.023)$. However, the genetic distance intergroup TS nLand TB $\mathrm{nL}(0.017)$ is smaller than that between TS L and TB L (0.025).

Table 6 reveals a no significant FST equal to 0 that can illustrate a very little differentiation between healthy people and affected populations. While values between populations divided according haplogroup, appear moderate and significant, with values existing between tissue groups from populations (TS L and TB L) belonging to

Table 3. Genetic diversity parameters of healthy tissue and benign tissue.

\begin{tabular}{ccc}
\hline & Healthy Tissue & Benign Tissue \\
\hline Sample size, N: & 29 & 28 \\
Length sequence without gaps, L: & 560 & 560 \\
Polymorphic sites, V: & $95(16.99 \%)$ & $68(12.14 \%)$ \\
Informative variable sites, Pi : & $28(29.47 \%)$ & $39(57.36 \%)$ \\
Total number of mutations, Eta: & $102(18.25 \%)$ & $73(13.06 \%)$ \\
Transitions. s: & $69.01 \%$ & $67.76 \%$ \\
Nature mutations & $30.99 \%$ & $32.24 \%$ \\
Transversion. v: & 2.267 & 2.049 \\
\hline
\end{tabular}

Table 4. Frequencies of nucleotide sequences.

\begin{tabular}{ccccccc}
\hline & $\mathrm{T} \%$ & $\mathrm{C} \%$ & $\mathrm{~A} \%$ & $\mathrm{G} \%$ & $(\mathrm{~T}+\mathrm{C}) \%$ & 55.00 \\
\hline Healthy Tissue & 24.99 & 30.01 & 27.44 & 17.56 & 45.00 & 55.07 \\
Benign Tissue & 24.95 & 30.12 & 27.69 & 17.24 & 44.93 \\
\hline
\end{tabular}

Table 5. Genetic distances of populations and population groups.

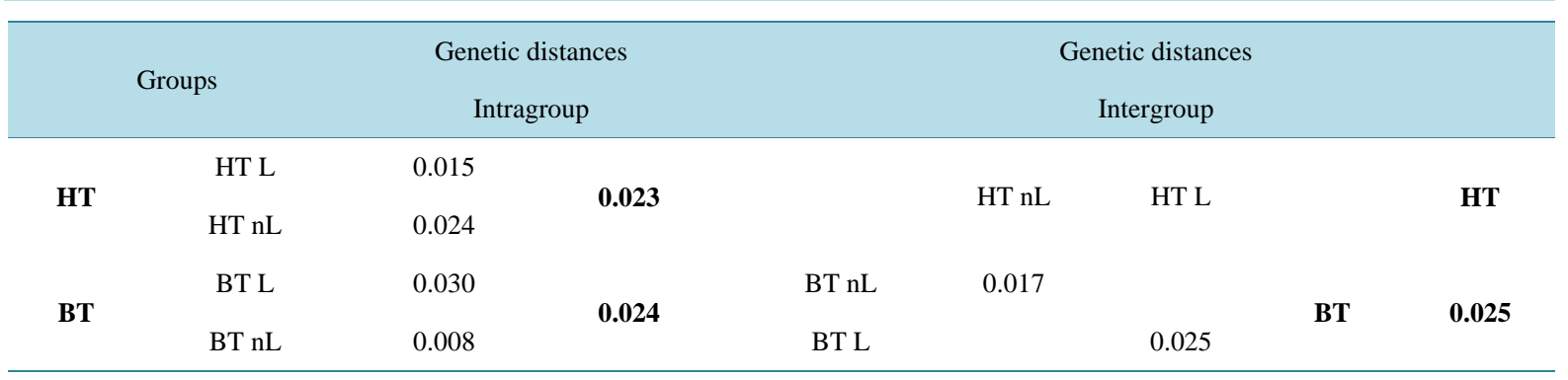

HT L: healthy tissue belonging to haplogroup L; BT nL: benign tissue not belonging to haplogroup L. 
Table 6. Values of genetic differentiation (FST) between populations and population groups.

\begin{tabular}{|c|c|c|c|c|}
\hline & HT & & HT L & $\mathrm{HT} n \mathrm{~L}$ \\
\hline BT & $\mathbf{0 . 0 0 0 0}(\mathrm{p}=0.991)$ & $\begin{array}{l}\text { BT L } \\
\text { BT nL }\end{array}$ & $0.077^{*}$ & \multirow{2}{*}{$0.067 *$} \\
\hline $\mathrm{TS}=\mathrm{H}$ & $\mathrm{TB}=$ Benign Tissue & & & \\
\hline
\end{tabular}

haplogroup L (FST $=0.077)$ and between groups populations of tissue (TS $\mathrm{nL}$ and $\mathrm{TB} \mathrm{nL}$ ) not belonging to haplogroup L (FST = 0.067) lower.

\section{3) Evolution of the D-loop}

A high haplotype diversity (Hd) and a low nucleotide diversity (Pi) were observed with 1 and 0.0238 for benign tissues, and 0.998 and 0.022 for healthy tissue, respectively. These results also show that the Hd and Pi indices benign tissue are slightly higher than those of healthy tissues. In Table 7 there are recorded values of genetic diversity indices (Hd and $\mathrm{Pi}$ ) TS and TB.

$\mathrm{D}$ Tajima $(-1.1134), \mathrm{D} *(-1.2926)$ and $\mathrm{F} *(-1.4558) \mathrm{Fu}$ and $\mathrm{Li}$, show for all benign tissue, negative and non-significant values. The $\mathrm{H}$ Fay and $\mathrm{Wu}$ is null and non-significant while the Fs of Fu (-18.22) is negatively significant (Table 8).

Graphical representations of the distribution of genetic distances between benign tissues of patients taken in pairs or Mismath distribution (Figure 1), according to the hypothesis of a constant population or growing population, show a multimodal distribution.

\subsubsection{Study on the Incidence of Benign Tumors}

Table 8 shows a significant difference in the presence of benign tumors, among the very young patients, aged under 20, whose age is known (38.09\%) of those at least 20 years (28 57\%), belonging to haplogroup L, while in the overall $52.38 \%(p=0.011)$ were 20 years or older. Among patients whose tumor type is known, $76.47 \%(p=$ 0.026) are suffering from fibroadenoma which $52.94 \%$ are of haplogroup L, with a significant presence of cystic tumors, epithelial, and HSTP good rating $(11.77 \%, \mathrm{p}<0.05)$ in patients of Asian haplogroup (B \& M).

For the microsatellite located between positions 303 and 316 of the noncoding region of the mtDNA molecule, three (3) haplotypes were observed in two patients and (2) in healthy subjects. A control sequence was obtained for Senegalese Women for D310 region. It consists of 7C in the first section and 6C in the second section. It is identified in almost all (96.55\%) healthy subjects in this study. Haplotype C309CC identified only in patients shows a very significant presence (with 35.71\%; $\mathrm{p}=6.42 \mathrm{e}-13$ ). Comparison of the haplotype frequencies between the control patients $(60.71 \%)$ and healthy subjects (96.55\%) gives a $\mathrm{p}$-value value highly significant ( $\mathrm{p}=$ $4.41 \mathrm{e}-11$ ) associated with an Odd ratio value equal to 0.0472. Haplotype C309T is carried by only one healthy individual, while haplotype C311T is present in a single patient. All these polymorphic differences noted in Table 9, are characterized by substitution of a base $\mathrm{C}$ by a T basis, except for the insertion C309CC.

\section{Discussion}

Mutations and polymorphisms of mtDNA have been the subject of intense research for over a decade, in an attempt to understand how they affect fundamental processes such as cancer and aging [20].

The D-loop is the primary replication site of control of mitochondrial DNA and its transcribed [21]. In several of these studies of mtDNA polymorphisms were mainly found in two hypervariable regions of the D-loop: HV1 (16024-16383) and HV2 (57-333) [22]. Indeed, among the changes reported in the literature during recent years, the D310, area located between nucleotides 303 and 315 and interrupted by a $\mathrm{T}$ at position 310, has been frequently studied in breast tumor samples [23]-[25]. And it was found that in approximately $42.18 \%$ of the region mutated [26].

This poly-C portion [27] (CCCCCCC..TCCCCC), involved in the formation of a persistent RNA-DNA hybrid, which led to the opening of mtDNA during replication of the strand heavy [28] [29], is highly polymorphic in particular the section between C-7 and C-9, with the most common sequence represented by the 7-C [30]-[32]. D310 some variations have also been reported as common variants in normal human tissues (Torroni et al., 1999). An in vitro analysis revealed that the D-loop, especially the D310, is more susceptible to oxidative damage and electrophilic attack compared to other regions of mtDNA [33]. 
Table 7. Haplotype diversity (Hd) and nucleotide (Pi) of healthy tissue and benign tissue.

\begin{tabular}{ccc}
\hline & Healthy Tissue & Benign Tissue \\
\hline Number of haplotypes, $\mathbf{h}$ & 28 & 28 \\
Haplotype diversity, $\mathbf{H d} \pm \mathbf{s d}$ & $0.998 \pm 0.01$ & $1 \pm 0.01$ \\
Nucleotide diversity, $\mathbf{P i} \pm \mathbf{s d}$ & $0.0220 \pm 0.0058$ & $0.0238 \pm 0.0031$ \\
\hline
\end{tabular}

Table 8. Tests of neutrality.

\begin{tabular}{cc}
\hline D de Tajima & Benign Tissue \\
P-value & -1.1134 \\
D* de Fu and Li & $\mathrm{p}>0.1$ \\
P-value & -1.2926 \\
F* de Fu and Li & $\mathrm{p}>0.1$ \\
P-value & -1.4558 \\
H de Fay and Wu & $\mathrm{p}>0.1$ \\
P-value & 0.00 \\
Fs de Fu & $\mathrm{p}>0.1$ \\
P-value & -18.22 \\
\hline
\end{tabular}

Table 9. Comparison between haplogroups D-loop, age of patients and types of benign tumors.

\begin{tabular}{|c|c|c|c|c|c|c|c|c|c|}
\hline \multirow{2}{*}{\multicolumn{2}{|c|}{ Haplogroups BT }} & \multicolumn{4}{|c|}{ Age } & \multicolumn{4}{|c|}{ Typology } \\
\hline & & \multirow{2}{*}{$\begin{array}{l}<20 \text { years } \\
8(38.09 \%)\end{array}$} & \multirow{2}{*}{$\begin{array}{l}\geq 20 \text { years } \\
6(28.57 \%)\end{array}$} & \multirow{2}{*}{$\frac{\mathrm{ND}}{4}$} & \multirow{2}{*}{$\begin{array}{l}\text { P-Value } \\
\mathrm{p}<0.05\end{array}$} & \multirow{2}{*}{$\begin{array}{c}\text { Fibroadenoma } \\
9(52.94 \%)\end{array}$} & \multirow{2}{*}{$\begin{array}{c}\text { Other } \\
2(11.77 \%)\end{array}$} & \multirow{2}{*}{$\begin{array}{c}\text { ND } \\
7\end{array}$} & \multirow{2}{*}{$\begin{array}{l}\text { P-Value } \\
\mathrm{p}>0.10\end{array}$} \\
\hline $\mathbf{L}$ & $64.29 \%$ & & & & & & & & \\
\hline B \& $\mathbf{M}$ & $32.14 \%$ & $2(9.52 \%)$ & 4 (19.04\%) & 3 & $\mathrm{p}>0.10$ & $3(17.65 \%)$ & $2(11.77 \%)$ & 3 & $\mathrm{p}<0.05$ \\
\hline $\mathbf{U}$ & $3.57 \%$ & - & $1(4.76 \%)$ & - & $0.05<\mathrm{p}<0.1$ & $1(5.88 \%)$ & - & - & $\mathrm{p}>0.10$ \\
\hline Total & $100 \%$ & $10(47.61 \%)$ & $11(52.38 \%)$ & 7 & 0.011 & $13(76.47 \%)$ & 4 (23.53\%) & 10 & 0.026 \\
\hline
\end{tabular}

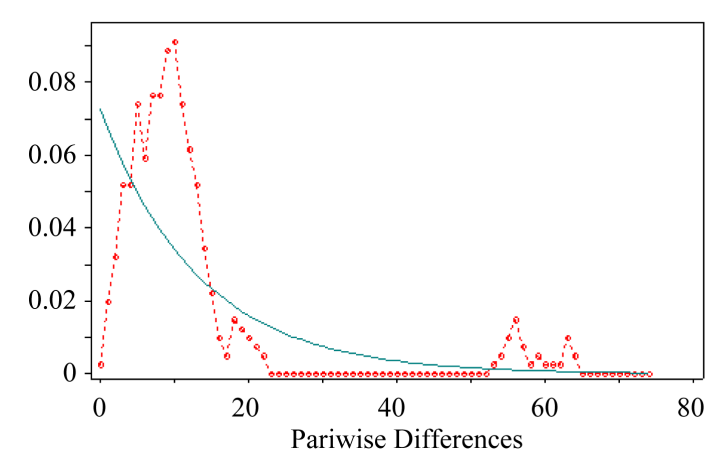

(a)

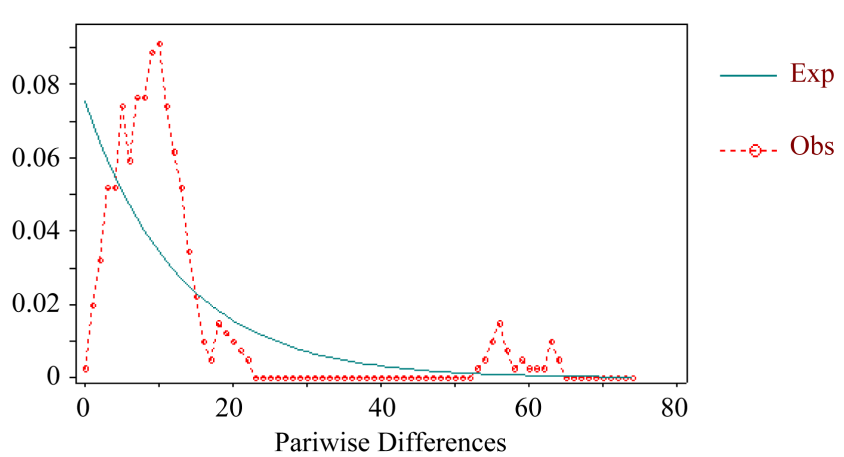

(b)

Figure 1. Curves mismatch distribution of the D-loop of benign tissue under the assumption of a constant population (a) and an expanding population (b). 
Table 10. Comparison of mitochondrial microsatellite haplotypes 303-316 (D310).

\begin{tabular}{|c|c|c|c|c|c|c|}
\hline Pattern & Sequence between 303 - 316 bp & HT (n = 29) & BT $(n=28)$ & P-value & OR & $95 \% \mathrm{CI}$ \\
\hline Witness & СССССССТСССССС & $28(96.55 \%)$ & $17(60.71 \%)$ & $* 4.41 \mathrm{e}-11$ & 0.0472 & $0.0140-0.1592$ \\
\hline C309CC & СССССССЕ్ТСССССС & - & $10(35.71 \%)$ & $* 6.42 \mathrm{e}-13$ & - & - \\
\hline C309T & ССССССТTСССССС & $1(3.45 \%)$ & - & 0.1213 & - & - \\
\hline C311T & СССССССТТССССС & - & $1(3.57 \%)$ & 0.1213 & - & - \\
\hline
\end{tabular}

Polymorphisms of mitochondrial DNA are widely used at present as molecular markers in evolutionary biology and population genetics in studies on the distribution of genetic variability levels within and between population-the relatedness between individuals, etc.

In addition to cytoplasmic localization of mitochondria and high copy number of mtDNA molecules, add its quasi-native transmissibility and replicative segregation experienced by mitochondria during cell divisions. Several recent studies of mtDNA in the tumor reported high mutation rate of the non-coding region of the D-loop.

The identification of these mutations of the mitochondrial DNA considered pathogenic in benign lesions, as well as their early onset, may be particularly interesting.

In this present study, a total of 28 patients with benign breast tumors and 29 control subjects were analyzed in order to determine the genetic diversity and evolution of the D-loop in benign tumors of the breast in Senegalese women. Among the more common variants, the mutations G16390A; T16519C; A73G; T146C; C182T; T195C; A263G; C315CC have less than 1 Odd ratio $(\mathrm{OR}<1)$ that mean a reduction in the relative risk RR, and therefore a preventive factor to the presence of benign tumors of the breast, with the exception of the T152C mutation more present in a non-significant way $(\mathrm{p}=0.9281)$ in patients $(78.57 \%)$ than in healthy subjects $(55.17 \%)$. Two common variants have also been identified, C150T, associated in some studies with longevity [34], more frequent significantly $\left(\mathrm{p}=1.01 \mathrm{e}^{-05}\right)$ in normal tissues than in benign tissues with an $\mathrm{OR}=0.3007$, unlike the G247A mutation ( $p=0.0486$, OR $=2.0798)$. And the C150T mutation could be associated with protection in the presence of benign breast tumor while the G247A mutation is involved in an increased risk. A very significant difference ( $<$ 2.32e-07) was observed for all the most common mutations specific to patients (G79T, G81T; G97A, G228A, T310C) compared to the control group. These can be found to be associated with a risk of benign breast tumor among Senegalese women, while C16478A mutations, C151G, T204G and G207A would be associated with an absence of tumor present in a significant way $(p<0.0002)$ in healthy subjects.

Many studies have identified several mutations detected in both the genes of the human mitochondrial DNA and in the main non-coding region (D-loop). In literature, some studies suggest the involvement of mutations of this mtDNA control region containing the main promoters in human carcinogenesis [35]-[37]. Several studies have even reported the presence of the mutations T146C, T152C, C150T and other polymorphisms in the tumor cells [38] [39] and in various body tissues and fluids [40]-[43]. The C150T mutation in particular was significantly indicated for an increased risk of cervical cancer among Chinese women [44] while T16519C is recognized to increase the risk of breast cancer [45]. Thus these results show that these specific mitochondrial polymorphisms may be useful for the development of biomarkers for early detection of tumors.

The study of patients with some of these mitochondrial mutations, was continued to distinguish haplogroups which are more apt of developing a benign breast tumor.

And a prevalence of the presence of benign tumors in, higher and significant $(\mathrm{p}=0.0104)$ with $\mathrm{OR}=2.1728$ in a confidence interval $95 \% \mathrm{CI}=(1.2320$ - 3.8322) for mitochondrial haplogroups d Sub-Saharan origin (mainly L) and a significantly lower prevalence $(\mathrm{p}=4.05 \mathrm{e}-07)$ with $\mathrm{OR}=0.0927$ in a $95 \% \mathrm{CI}=(0.0313$ 0.2748) for the origins of European haplogroups and Berber, have been noticed in patients compared to controls group. Despite the non-significant higher prevalence observed for haplogroups Asian origin, one is tempted to say that the more Senegalese women's breast tissue are from $\mathrm{L}$ mitochondrial haplogroup, the higher is the risk of getting benign breast tumor , while haplogroups ( $\mathrm{H}$ and $\mathrm{T}$ in particular ) of Berber and European origins have a protective effect in this study population.

The mitochondrial haplogroup composition of this population revealed a broad spectrum of different geographical origins haplogroups. It turned out that in the first human settlements, people from Europe and Asia 
have brought with them a fraction of their genetic variability, leaving appear in SSA non-African haplogroups [46]-[49], as demonstrated in this study of Senegalese population. In fact, Chen et al. [17] in a study of mtDNA variation in African populations, remarked that "in addition to haplogroup L, other haplogroups that were more similar to those noted in the European and Asian were present in Senegal”. It is because of the long cultural and economic interactions that occurred with saharan and north african people, and genetic exchanges with European populations. However, according Torroni et al. [50]-[53], African haplogroup L is the most divergent and the oldest to the specific haplogroups regarding the Asian, European and Native American. Thus this high prevalence recorded for subjects belonging to haplogroup L may suggest that it may be the result of this high divergence. Studying these different variants observed today in these different haplogroups and sub haplogroups, particularly those of the line L, will allow certainly to better identify the mutations responsible for benign breast disease, but also to understand the evolution history of senegalese people.

To examine the effect of these evolutionary forces in this studied population, several parameters were investigated. Thus, it has been noticed in the results, the parsimony informative sites as well as the number of transversions are higher in TB than in TS. This leads to a value of the genetic distance intra higher benign tissue at the level of TS.

The FST obtained allowed also to observe a moderate genetic differentiation between populations (TS L and $\mathrm{L} \mathrm{TB}$ ) belonging to haplogroup $\mathrm{L}(\mathrm{FST}=0.077)$ and that between populations ( $\mathrm{TS} \mathrm{nL}$ and $\mathrm{TB} \mathrm{nL}$ ) not belonging to haplogroup L (FST $=0.067)$. These results correlated with the values of genetic distances inter populations belonging to haplogroup $L(d=0.025)$ and between populations that do not belong to haplogroup $L(d=0.017)$ illustrate the existence of a difference Genetic between healthy tissue and benign tumors, but also supports the fact that the risk of benign breast tumor is associated with Senegalese women whose mitochondrial haplogroup is L, which have a genetic distance inter populations and higher TSP than those observed for people not belonging to haplogroup $\mathrm{L}$.

The analysis of the genetic variability of the D-loop of breast tissues showed high haplotype diversity (Hd) and low nucleotide diversity (Pi), both in benign tissue than in control tissue with a slight superiority $\mathrm{Hd}$ and $\mathrm{Pi}$ clues in TB. This would suggest a rapid growth of breast cells, more accentuated in benign breast tumors. Typically the tumor cells are characterized by a more rapid cell proliferation, due to the presence of alterations in genes that regulate the proliferation in normal cells (or proto-oncogenes), which is then activated in oncogenes and suppressor genes tumor (or anti-oncogenes), associated with dysfunction of the DNA opposite the regulation of the cell cycle and genome repair system [54] [55]. These are actually mutations which are the ultimate source of genetic variation at the origin of diversity for all organisms, thus playing the role of engine of evolution [56] [57]. However, according to the model of Motoo Kimura called "theory of mutation and random drift": most of the genetic variability is neutral and polymorphisms are removed or fixed in people under the influence of effects environmental genetic drift [58].

In order to estimate the influence of neutral processes and selective processes, the study of the evolution of the D-loop in Senegalese patients has been continued. And the results show for negative values of neutrality and non-significant tests of Tajima $\mathrm{D}, \mathrm{D} *$ and $\mathrm{F} * \mathrm{Fu}$ and $\mathrm{Li}$, rejecting then the idea of a possible selection of the D-loop. This trend was confirmed by the Fu' Fs value that is significantly negative, as the H Fay and Wu which turned null and unsignificant. Indeed for non-coding sequences such as mitochondrial DNA control region, a gap regarding neutrality is most likely explained by recent demographic changes than by selection [59], in other words this evolution would be due to an excess of recent changes, the cause of this presence of benign legions in breast. These findings on the D-loop's evolution seem to confirm the non-lethal benign tumors unlike cancerous tumors for which it has been shown that D-Loop is under selection [60]. The curves of mismatch distributions that have shown a multimodal distribution for benign tissue with a deviation of the observed distribution compared to that expected, suggest the signing of a moderate growth of a given population.

In the study on the incidence of benign breast tumors in Senegalese women, the linkage of haplogroups and clinic pathological parameters in this case the age and typology found in patients of haplogroup L, a significant prevalence for younger patients, aged under 20, while overall a significant prevalence was observed for patients aged 20 years or more. Furthermore a significant incidence of fibroadenomas was observed among benign breast present in this sample.

The analysis of microsatellite polymorphism located between positions 303 and 316 of the D-loop, found that of all 28 patients studied, 17 (or $60.71 \%$ ) have in common haplotype indicator with the majority healthy individuals (96.55\%) and almost one third of patients (35.71\%) have specific haplotype C309CC to TB. After com- 
parison of the distribution of haplotype frequencies between the carriers of benign tumors in healthy women and a highly significant $\mathrm{p}$-value value $(\mathrm{p}=4.41 \mathrm{e}-11)$ associated with a value of 0.0472 was $\mathrm{OR}=$ the witness observed haplotype and for the reason C309CC a very significant presence ( $\mathrm{p}=6.42 \mathrm{e}-13)$ was noted. These correlate results would mean that among the Senegalese with haplotype witness, nearly $2 / 3$ are likely to get a breast tumor while those with haplotype C309CC would constitute an increased risk group. However, the study of a large group of patients and controls should be considered to confirm these results.

\section{Conclusions}

Examining the process of tumorigenesis by molecular analysis of mtDNA remains at present a great challenge because many factors must be taken into account, increasing the complexity and difficulties of analysis and interpretation of results.

With the aim to establish a correlation between changes in the D-loop of mtDNA and the appearance among Senegalese women of benign tumors, we observed initially a genetic diversity of the population assigned with respect to the normal. Thus, in addition to the big haplotype diversity recorded for all individuals studied, the results revealed the existence of a genetic difference between healthy tissue and benign tumors, and a significant presence of specific variants with benign breast tumors, as well as healthy tissue.

Therefore we can state that the D-loop is a good molecular marker for early and specific detection of benign and precancerous lesions.

\section{References}

[1] Ly, M., Antoine, M., André, F., Callard, P., Bernaudin, J.F. and Diallo, D.A. (2011) Le cancer du sein chez la femme de l'Afrique sub-saharienne: Etat actuel des connaissances. Bulletin du Cancer, 98, 797-806.

[2] Badid, N. (2012) Stress oxydatif et profil nutritionnel chez une population de femmes atteintes de cancer du sein dans la région de Tlemcen. Thèse Université Abou Bekr Belkaid Tlemcen, Algérie, 200 p.

[3] Boisvert, G. (2013) Les lésions bénignes du sein. Spécial cancer du sein. Le patient, 7, 38-41.

[4] Chauvet, M.P., Jafari, M., Lambaudie, E. and Giard, S. (2006) Benign Tumors and Complex Lesions: Surgeon’s Point of View. 28èmes journées de la Société française de sénologie et de pathologie mammaire (SFSPM), Lille, 355-360.

[5] www.lemonde.fr

[6] www.novartis.fr/

[7] Picard, S.F. (2003) Rôle de l'instabilité génétique dans la réponse aux traitements dans une population de cancers du sein localement avancés et traités par chimiothérapie première. Mémoire école pratique des hautes études, France, 41 p.

[8] www.larousse.fr

[9] Mbaye, F., Dem, A., Fall, M. and Sembène, M. (2012) Implication of the Cytochrome Bull Nucleotide and Protein Mutations in the Occurrence of Breast Cancer in Senegal. International Journal of Applied Biology and Pharmaceutical Technology, 3, 107-114.

[10] Hall, T. (2001) BioEdit Version 5.0.6. North Carolina State University, Raleigh.

[11] Thompson, J.D., Higgins, D.G. and Gibson, T.J. (1994) CLUSTAL W: Improving the Sensitivity of Progressive Multiple Sequence Alignment through Sequence Weighting, Position-Specific Gap Penalties and Weight Matrix Choice. Nucleic Acids Research, 22, 4673-4680. http://dx.doi.org/10.1093/nar/22.22.4673

[12] Long, F. and Yong-Gang, Y. (2013) An Update to MitoTool: Using a New Scoring System for Faster mtDNA Haplogroup Determination. Mitochondrion, 13, 360-363. http://dx.doi.org/10.1016/j.mito.2013.04.011

[13] www.mitomap.org/mitoseq.html.

[14] Coudray, C., Torroni, A., Achilli, A., Pala, M., Olivieri, A., Larrouy, G. and Dugoujon, J.M. (2009) Les lignées mitochondriales et l'histoire génétique des populations berbérophones du nord de l’Afrique. Antropo, 18, 63-72. www.didac.ehu.es/antropo

[15] Tamura, K., Stecher, G., Peterson, D., Filipski, A. and Kumar, S. (2013) MEGA 6: Molecular Evolutionary Genetics Analysis Using Maximum Likelihood, Evolutionary Distance, and Maximum Parsimony Methods. Molecular Biology and Evolution, 30, 2725-2729. http://dx.doi.org/10.1093/molbev/mst197

[16] Excoffier, L., Laval, G. and Schneider, S. (2005) Arlequin Version 3.0: An Integrated Software Package for Population Genetics Data Analysis. Evolutionary Bioinformatics Online, 1, 47-50.

[17] Chen, Y.-S., Torroni, A., Excoffier, L., Santachiara-Benerecetti, A.S. and Wallace, D.C. (1995) Analysis of mtDNA 
Variation in African Populations Reveals the Most Ancient of All Human Continent Specific Haplogroups. American Journal Human Genetics, 57, 133-149.

[18] Rozas, J., Librado, P., Sánchez-Del Barrio, J.C., Messeguer, X. and Rozas, R. (2010) DnaSP Version 5 Help Contents (Help File). http://www.ub.edu/dnasp/

[19] www.xlstat.com.

[20] Czarnecka, A.M. and Bartnik, E. (2011) The Role of the Mitochondrial Genome in Ageing and Carcinogenesis. Journal of Aging Research, 2011, Article ID: 136435.

[21] Suzuki, M., Toyooka, S., Miyajima, K., Iizasa, T., Fujisawa, T., Bekele, N.B. and Gazdar, A.F. (2003) Alterations in the Mitochondrial Displacement Loop in Lung Cancers. Clinical Cancer Research, 9, 5636-5641.

[22] Klemba, A., Kowalewska, M. and Kukwa, W. (2010) Mitochondrial Genotype in Vulvar Carcinoma-Cuckoo in the Nest. Journal of Biomedical Science, 17, 73. http://dx.doi.org/10.1186/1423-0127-17-73

[23] Parrella, P., Seripa, D., Matera, M.G., Rabitti, C., Rinaldi, M., Mazzarelli, P., Gravina, C., Gallucci, M., Altomare, V., Flammia, G., Casalino, B., Benedetti-Panici, P.L. and Fazio, V.M. (2003) Mutations of the D310 Mitochondrial Mononucleotide Repeat in Primary Tumors and Cytological Specimens. Cancer Letters, 190, 73-77. http://dx.doi.org/10.1016/S0304-3835(02)00578-5

[24] Aral, C., Kaya, H., Ataizi-Celikel, C., Akkiprik, M., Sonmez, O., Gulluoglu, B.M. and Ozer, A. (2006) A Novel Approach for Rapid Screening of Mitochondrial D310 Polymorphism. BMC Cancer, 6, 21.

[25] Xu, C., Tran-Thanh, D., Ma, C., May, K., Jung, J., Vecchiarelli, J. and Done, S.J. (2012) Mitochondrial D310 Mutations in the Early Development of Breast Cancer. British Journal of Cancer, 106, 1506-1511. http://dx.doi.org/10.1038/bjc.2012.74

[26] Santos Jr., G.C., Góoes, A.C., Vitto, H.D., Moreira, C.C., Avvad, E., Rumjanek, F.D. and Gallo, C.V. (2012) Genomic Instability at the 13q31 Locus and Somatic mtDNA Mutation in the D-Loop Site Correlate with Tumor Aggressiveness in Sporadic Brazilian Breast Cancer Cases. Clinics (Sao Paulo), 67, 1181-1190. http://dx.doi.org/10.6061/clinics/2012(10)10

[27] Yacoubi-Loueslati, B., Troudi, W., Baccar, A., Cherni, L., Ben Rhomdhane, K. and Ben Ammar Elgaaied, A. (2009) Polymorphism of the Mitochondrial Microsatellite 303-315 in Breast Cancer in Tunisia. Bulletin du Cancer, 96, 337342.

[28] Kang, D., Miyako, K., Kai, Y., Irie, T. and Takeshige, K. (1997) In Vivo Determination of Replication Origins of Human Mitochondrial DNA by Ligation-Mediated Polymerase Chain Reaction. Journal of Biology and Chemistry, 272, 15275-15279. http://dx.doi.org/10.1074/jbc.272.24.15275

[29] Lee, D.Y. and Clayton, D.A. (1998) Initiation of Mitochondrial DNA Replication by Transcription and R-Loop Processing. Journal of Biology and Chemistry, 273, 30614-30621. http://dx.doi.org/10.1074/jbc.273.46.30614

[30] Jin, X., Zhang, J., Gao, Y., Ding, K., Wang, N., Zhou, D., Jen, J. and Cheng, S. (2007) Relationship between Mitochondrial DNA Mutations and Clinical Characteristics in Human Lung Cancer. Mitochondrion, 7, 347-353. http://dx.doi.org/10.1016/j.mito.2007.06.003

[31] Yu, M., Zhou, Y., Shi, Y., Ning, L., Yang, Y., Wei, X., Zhang, N., Hao, X. and Niu, R. (2007) Reduced Mitochondrial DNA Copy Number Is Correlated with Tumor Progression and Prognosis in Chinese Breast Cancer Patients. International Union of Biochemistry and Molecular Biology Life, 59, 450-457. http://dx.doi.org/10.1080/15216540701509955

[32] Chatterjee, A., Dasgupta, S. and Sidransky, D. (2011) Mitochondrial Subversion in Cancer. Cancer Prevention Research, 4, 638-654. http://dx.doi.org/10.1158/1940-6207.CAPR-10-0326

[33] Mambo, E., Gao, X., Cohen, Y., Guo, Z., Talalay, P. and Sidransky, D. (2003) Electrophile and Oxidant Damage of Mitochondrial DNA Leading to Rapid Evolution of Homoplasmic Mutations. Proceedings of the National Academy of Sciences of the United States of America, 100, 1838-1843. http://dx.doi.org/10.1073/pnas.0437910100

[34] Chen, A., Raule, N., Chomyn, A. and Attardi, G. (2012) Decreased Reactive Oxygen Species Production in Cells with Mitochondrial Haplogroups Associated with Longevity. PLoS ONE, 7, e46473. http://dx.doi.org/10.1371/journal.pone.0046473

[35] Lee, H.-C., Li, S.H., Lin, J.C., Wu, C.C., Yeh, D.C. and Wei, Y.-H. (2004) Somatic Mutations in the D-Loop and Decrease in the Copy Number of Mitochondrial DNA in Human Hepatocellular Carcinoma. Mutation Research, 547, 7178. http://dx.doi.org/10.1016/j.mrfmmm.2003.12.011

[36] Van Trappen, P.O., Cullup, T., Troke, R., Swann, D., Shepherd, J.H., Jacobs, I.J., Gayther, S.A. and Mein, C.A. (2007) Somatic Mitochondrial DNA Mutations in Primary and Metastatic Ovarian Cancer. Gynecologic Oncology, 104, 129133. http://dx.doi.org/10.1016/j.ygyno.2006.07.010

[37] Sharawat, S.K., Bakhshi, R., Vishnubhatla, S. and Bakhshi, S. (2010) Mitochondrial D-Loop Variations in Paediatric 
Acute Myeloid Leukaemia: A Potential Prognostic Marker. British Journal of Haematology, 149, 391-398. http://dx.doi.org/10.1111/j.1365-2141.2010.08084.x

[38] Chen, J.Z. and Kadlubar, F.F. (2004) Mitochondrial Mutagenesis and Oxidative Stress in Human Prostate Cancer. Journal of Environmental Science and Health, Part C: Environmental Carcinogenesis \& Ecotoxicology Reviews, 22, 112. http://dx.doi.org/10.1081/GNC-120037931

[39] Yoneyama, H., Hara, T., Kato, Y., Yamori, T., Matsuura, E.T. and Koike, K. (2005) Nucleotide Sequence Variation Is Frequent in the Mitochondrial DNA Displacement Loop Region of Individual Human Tumor Cells. Molecular Cancer Research, 3, 14-20.

[40] Fliss, M.S., Usadel, H., Caballero, O.L., Wu, L., Buta, M.R., Eleff, S.M., Jen, J. and Sidransky, D. (2000) Facile Detection of Mitochondrial DNA Mutations in Tumors and Bodily Fluids. Science, 287, 2017-2019. http://dx.doi.org/10.1126/science.287.5460.2017

[41] Jerónimo, C., Nomoto, S., Caballero, O.L., Usadel, H., Henrique, R., Varzim, G., Oliveira, J., Lopes, C., Fliss, M.S. and Sidransky, D. (2001) Mitochondrial Mutations in Early Stage Prostate Cancer and Bodily Fluids. Oncogene, 20, 5195-5198. http://dx.doi.org/10.1038/sj.onc.1204646

[42] Zhu, W., Qin, W., Wessel, A., Puckett, C.L. and Sauter, E. (2005) Mitochondrial DNA Mutations in Breast Cancer Tissue and in Matched Nipple Aspirate Fluid. Carcinogenesis, 26, 145-152. http://dx.doi.org/10.1093/carcin/bgh282

[43] Wang, C., Zhang, F., Fan, H., Peng, L., Zhang, R., Liu, S. and Guo, Z. (2011) Sequence Polymorphisms of Mitochondrial D-Loop and Hepatocellular Carcinoma Outcome. Biochemical and Biophysical Research Communications, 406, 493-496. http://dx.doi.org/10.1016/j.bbrc.2011.02.088

[44] Zhai, K., Chang, L., Zhang, Q., Liu, B. and Wu, Y. (2011) Mitochondrial C150T Polymorphism Increases the Risk of Cervical Cancer and HPV Infection. Mitochondrion, 11, 559-563. http://dx.doi.org/10.1016/j.mito.2011.02.005

[45] Bai, R.K., Leal, S.M., Covarrubias, D., Liu, A. and Wong, L.J. (2007) Mitochondrial Genetic Background Modifies Breast Cancer Risk. Cancer Research, 67, 4687-4694. http://dx.doi.org/10.1158/0008-5472.CAN-06-3554

[46] Cann, R.L., Stoneking, M. and Wilson, A.C. (1987) Mitochondrial DNA and Human Evolution. Nature, 325, 31-36. http://dx.doi.org/10.1038/325031a0

[47] Excoffier, L. (2002) Human Demographic History: Refining the Recent African Origin Model. Current Opinion in Genetics and Development, 12, 675-682. http://dx.doi.org/10.1016/S0959-437X(02)00350-7

[48] Jakobsson, M., Scholz, S.W., Scheet, P., Gibbs, J.R., VanLiere, J.M., Fung, H.C., Szpiech, Z.A., Degnan, J.H., Wang, K., Guerreiro, R., Bras, J.M., Schymick, J.C., Hernandez, D.G., Traynor, B.J., Simon-Sanchez, J., Matarin, M., Britton, A., Van De Leemput, J., Rafferty, I., Bucan, M., Cann, H.M., Hardy, J.A., Rosenberg, N.A. and Singleton, A.B. (2008) Genotype, Haplotype and Copy-Number Variation in Worldwide Human Populations. Nature, 451, 998-1003. http://dx.doi.org/10.1038/nature06742

[49] Li, J.Z., Absher, D.M., Tang, H., Southwick, A.M., Casto, A.M., Ramachandran, S., Cann, H.M., Barsh, G.S., Feldman, M., Cavalli-Sforza, L.L. and Myers, R.M. (2008) Worldwide Human Relationships Inferred from Genome-Wide Patterns of Variation. Science, 319, 1100-1104. http://dx.doi.org/10.1126/science.1153717

[50] Torroni, A., Chen, Y.-S., Semino, O., Santachiara-Benerecetti, A.S., Scott, R.C., Lott, M.T., Winter, M. and Wallace D.C. (1994) Mitochondrial DNA and Y-Chromosome Polymorphisms in Four Native American Populations from Southern Mexico. The American Journal of Human Genetics, 54, 303-318.

[51] Torroni, A., Lott, M.T., Cabell, M.F., Chen, Y.-S., Lavergne, L. and Wallace, D.C. (1994) Mitochondrial DNA and the Origin of Caucasians: Identification of Ancient Caucasian-Specific Haplogroups, One of Which Is Prone to a Recurrent Somatic Duplication in the D-Loop Region. The American Journal of Human Genetics, 55, 760-776.

[52] Torroni, A., Miller, J.A., Moore, L.G., Zamudio, S., Zhuang, J., Droma, T. and Wallace, D.C. (1994) Mitochondrial DNA Analysis in Tibet. Implications for the Origin of the Tibetan Population and Its Adaptation to High Altitude. American Journal of Physical Anthropology, 93, 189-199. http://dx.doi.org/10.1002/ajpa.1330930204

[53] Torroni, A., Neel, J.V., Barrantes, R., Schurr, T.G. and Wallace, D.C. (1994) A Mitochondrial DNA “Clock” for the Amerinds and Its Implications for Timing Their Entry into North America. Proceedings of the National Academy of Sciences of the USA, 91, 1158-1162. http://dx.doi.org/10.1073/pnas.91.3.1158

[54] Poehlmann, A. and Roessner, A. (2010) Importance of DNA Damage Checkpoints in the Pathogenesis of Human Cancers. Pathology Research and Practice, 206, 591-601. http://dx.doi.org/10.1016/j.prp.2010.06.006

[55] Hanahan, D. and Weinberg, R.A. (2011) Hallmarks of Cancer: The Next Generation. Cell, 144, 646-674. http://dx.doi.org/10.1016/j.cell.2011.02.013

[56] Parent, G.J. (2008) Acclimatation thermique de la palourde américaine, Mercenaria mercenaria: Expérimentation en jardin commun. Mémoire Université du Québec à Rimouski, 76 p.

[57] William, M. (2011) Diversité génétique et adaptation au milieu chez les arbres forestiers tropicaux: Etude chez le genre Virola (Myristicaceae). Thèse génétique écologique, Université des Antilles et de la Guyane, Guadeloupe, 178 p. 
[58] Bergeault, K. (2010) Identification de deux genes NPR1 chez les Vitaceae, analyse de leur diversite de sequences et interactions avec les facteurs de transcription VVTGA. Thèse, Universite de Haute-Alsace, Mulhouse, 173 p.

[59] Razafindrazaka, H. (2010) Le peuplement humain de Madagascar: Anthropologie génétique de trois groupes traditionnels. Thèse Biotechnologie, Anthropobiologie, Université Toulouse III-Paul Sabatier, Toulouse, 277 p.

[60] Mbaye, F. (2015) Le cancer du sein chez les femmes senegalaises: Impact de la variabilite nucleotidique et de l’instabilite de loci microsatellites. Thèse Génétique des Populations, Universite Cheikh Anta Diop de Dakar, Dakar, $131 \mathrm{p}$. 